\title{
Online Vapors Separation of Torrefied-Bagasse/HDPE Co-pyrolysis into Three Grades of Bio-oils
}

Peter Keliona Wani Likun, ${ }^{\dagger} *$ Huiyan Zhang ${ }^{*},{ }^{\dagger}$ and Rui Xiao ${ }^{\dagger}$

$\dagger$ Key Laboratory of Thermal Energy Conversion and Control Ministry of Education, school of Energy and Environment, Southeast University, Nanjing, 210096, China

* Department of Mechanical Engineering, University of Juba, Juba, P.O. Box 82, South Sudan

*Tel.: +86-25-83795726. Fax: +86-25-83790667 Email: hyzhang@ @seu.edu.cn

The chemical compounds in the high-density polyethylene (HDPE) plastic oil identified by GC/MS.

\section{Supporting Information:}

Table S1 Detected compounds in the HDPE oil produced via pyrolysis

\begin{tabular}{ll}
\hline compound & area\% \\
\hline alkanes and derivatives & \\
\hline cyclohexane, methyl- & 0.52 \\
hexane, 2-methyl- & 1.74 \\
hexane, 3-methyl- & 1.12 \\
cyclopentane, 1,3-dimethyl-, cis- & 0.79 \\
cyclopentane, 1,3-dimethyl- & 1.28 \\
2-Pentene, 2,3-dimethyl- & 0.80 \\
cyclopentane, ethyl- & 0.76 \\
cyclopentane, 1,2,4-trimethyl- & 0.20 \\
cyclobutane, (1-methylethylidene)- & 2.02 \\
heptane, 2-methyl- & 0.33 \\
heptane, 3-methyl- & 0.43 \\
cyclopentane, ethylidene- & 0.26 \\
cyclopentane, 1-ethyl-3-methyl- & 0.63 \\
cyclohexane, 1,2-dimethyl-, cis- & 0.86 \\
cyclohexane, 1-methyl-4-methylene- & 0.63 \\
cyclopentane, 2-ethylidene-1,1-dimethyl- & 0.41 \\
cyclopropane, 1-methyl-2-(1-methylethyl)-3-(1- & 0.12 \\
methylethylidene)-, cis- & \\
cyclohexane, 1-methyl-4-(1-methylethenyl)-, trans- & 0.36 \\
cyclopentane, 1-methyl-1-(2-methyl-2-propenyl)- & 0.10 \\
decane, 3-methyl- & 0.09 \\
indane & 0.46 \\
hexane, 2,3,4-trimethyl- & 0.07 \\
cyclohexadecane & 0.05 \\
eicosane & 0.45 \\
tetracosane & 0.20
\end{tabular}




\begin{tabular}{|c|c|}
\hline octadecane & 0.16 \\
\hline subtotal & 14.83 \\
\hline aromatics \& derivatives & \\
\hline cyclohexene & 2.73 \\
\hline benzene & 5.91 \\
\hline cyclopentene, 4,4-dimethyl- & 2.53 \\
\hline 3-methyl-3-hexene & 0.74 \\
\hline cyclopentene, 1,5-dimethyl- & 1.62 \\
\hline cyclohexene, 1-methyl- & 0.44 \\
\hline cyclohexene, 4-methyl- & 0.36 \\
\hline 1,3-dimethyl-1-cyclohexene & 0.47 \\
\hline 1-ethylcyclopentene & 1.42 \\
\hline cyclopentene, 1,2,3-trimethyl- & 0.98 \\
\hline 1,3-cyclopentadiene, 1,2-dimethyl- & 0.27 \\
\hline cyclohexene, 3-methyl- & 1.39 \\
\hline 1,3-cyclopentadiene, 5,5-dimethyl- & 0.23 \\
\hline 1,3-dimethyl-1-cyclohexene & 2.29 \\
\hline toluene & 4.27 \\
\hline 1,3,5-cycloheptatriene & 4.19 \\
\hline cyclohexene, 3,5-dimethyl- & 1.81 \\
\hline 1,4-Hexadiene, 3-ethyl- & 0.41 \\
\hline methyl ethyl cyclopentene & 0.35 \\
\hline cyclohexene, 3-ethyl- & 0.32 \\
\hline 5,5-dimethyl-1,3-hexadiene & 0.44 \\
\hline trans-3,5-dimethylcyclohexene & 0.45 \\
\hline cyclohexane, 1-methyl-4-methylene- & 0.63 \\
\hline methyl ethyl cyclopentene & 0.64 \\
\hline 2,4-heptadiene, 2,6-dimethyl- & 0.95 \\
\hline cis-4-nonene & 0.25 \\
\hline cyclopentadiene, 2,5,5-trimethyl- & 0.76 \\
\hline 6,6-dimethylhepta-2,4-diene & 0.64 \\
\hline 2,4-hexadiene, 2,5-dimethyl- & 0.50 \\
\hline 4-nonene & 0.22 \\
\hline cyclopentene, 3-ethylidene-1-methyl- & 0.37 \\
\hline cyclopentane, 2-ethylidene-1,1-dimethyl- & 0.18 \\
\hline 1,1-dimethyl-4-methylenecyclohexane & 0.47 \\
\hline cyclohexene,1-propyl- & 0.24 \\
\hline ethylbenzene & 4.02 \\
\hline 3-heptene, 2,6-dimethyl- & 0.23 \\
\hline 2-methyl-2-octene & 0.26 \\
\hline p-xylene & 5.11 \\
\hline o-xylene & 3.22 \\
\hline 4-decene & 0.15 \\
\hline 1-methylcyclooctene & 0.38 \\
\hline 3-octyne, 2-methyl- & 0.27 \\
\hline benzene, 1,3-dimethyl- & 3.40 \\
\hline styrene & 0.12 \\
\hline cyclohexene, 1-methyl-4-(1-methylethyl)- & 0.17 \\
\hline cyclohexene, 3,3,5-trimethyl- & 0.24 \\
\hline cyclohexene, 3-methyl-6-(1-methylethyl)- & 0.09 \\
\hline cyclohexene,4-butyl- & 0.12 \\
\hline benzene, (1-methylethyl)- & 0.36 \\
\hline
\end{tabular}


cis-3-decene $\quad 0.22$

cis-4-decene 0.11

benzene, propyl- $\quad 0.64$

trans-4-decene 0.23

benzene, 1-ethyl-3-methyl- $\quad 6.18$

5-decene 0.14

mesitylene 0.52

5-undecene 0.17

benzene, 1-ethyl-2-methyl- $\quad 0.58$

benzene, 1,2,4-trimethyl- $\quad 2.07$

benzene, 1-methyl-2-propyl- $\quad 0.13$

benzene, 1-ethenyl-4-methyl- $\quad 0.10$

benzene, cyclopropyl- $\quad 0.14$

p-cymene 0.32

o-cymene 0.24

1-octene 0.11

5-undecene, $(E)$ - $\quad 0.08$

benzene, 1,2,3-trimethyl- $\quad 0.21$

benzene, 1,4-diethyl- $\quad 0.54$

benzene, 1-methyl-3-propyl- $\quad 0.66$

benzene, (1-methylpropyl)- $\quad 0.92$

benzene, 2-ethyl-1,4-dimethyl- $\quad 0.72$

3-undecene, $(Z)$ - 0.04

benzene, 1,3-diethyl- $\quad 0.05$

3-dodecene, $(E)$ - $\quad 0.08$

indene 0.11

benzene, 1-methyl-4-propyl- $\quad 0.10$

benzene, 4-ethyl-1,2-dimethyl- $\quad 0.20$

benzene, 2-ethyl-1,3-dimethyl- $\quad 0.17$

benzene, 1-ethyl-2,4-dimethyl- $\quad 0.69$

benzene, 1-ethenyl-3-ethyl- 0.21

benzene, 2,4-diethyl-1-methyl- $\quad 0.19$

benzene, (1,1-dimethylpropyl)- $\quad 0.14$

benzene, 1,3-dimethyl-5-(1-methylethyl)- $\quad 0.15$

benzene, 1-methyl-3-(1-methylethyl)- $\quad 0.12$

benzene, 1,2,4,5-tetramethyl- $\quad 0.20$

benzene, 1,2,3,4-tetramethyl- $\quad 0.21$

benzene, 1-ethyl-3,5-dimethyl- $\quad 0.19$

benzene, 1,3-diethyl-5-methyl- 0.08

benzene, 2-ethyl-1,4-dimethyl- $\quad 0.20$

$1 H$-indene, 2,3-dihydro-4-methyl- $\quad 0.40$

1-dodecene 0.37

benzene, 1-ethyl-2,3-dimethyl- $\quad 0.11$

4-dodecene 0.03

benzene, 1,2,4,5-tetramethyl- $\quad 0.12$

benzene, (1,1-dimethylpropyl)- $\quad 0.39$

benzene, 1,2-diethyl-3,4-dimethyl- $\quad 0.09$

$1 H$-indene, 2,3-dihydro-4,7-dimethyl- $\quad 0.26$

$1 H$-indene, 2,3-dihydro-1,6-dimethyl- $\quad 0.45$

benzene, pentamethyl- 0.13

benzene, 1-(2-butenyl)-2,3-dimethyl- 0.18

$1 H$-indene, 2,3-dihydro-1,2-dimethyl- $\quad 0.07$

benzene, 1-ethyl-4-(1-methylethyl)- $\quad 0.08$ 
benzene, 1-ethyl-3-(1-methylethyl)- 0.11

$1 H$-indene, 2,3-dihydro-1,1,3-trimethyl- $\quad 0.03$

6-tridecene, $(Z)$ - 0.04

benzene, (2-methyl-1-butenyl)- $\quad 0.12$

3-tridecene, $(E)$ - 0.14

5-tetradecene, $(E)$ - $\quad 0.09$

1-tridecene 0.26

$1 H$-indene, 2,3-dihydro-1,3-dimethyl- $\quad 0.04$

3-tridecene, $(Z)$ - 0.04

$1 H$-indene, 2,3-dihydro-1,5,7-trimethyl- $\quad 0.03$

benzene, 1,2-diethyl-3,4-dimethyl- $\quad 0.04$

$1 \mathrm{H}$-indene, 1,3-dimethyl- $\quad 0.15$

2-tetradecene, $(E)$ - $\quad 0.05$

7-tetradecene, $(E)$ - $\quad 0.05$

tetradecane 0.24

4-tetradecene, $(E)$ - $\quad 0.05$

1,2,3-trimethylindene $\quad 0.08$

1-pentadecene 0.02

7-hexadecene, $(Z)-\quad 0.05$

5-octadecene, $(E)$ - $\quad 0.03$

2-methyl-Z-4-tetradecene $\quad 0.02$

cetene 0.03

3-hexadecene, $(Z)$ - $\quad 0.01$

7-hexadecene, $(Z)-\quad 0.02$

1-nonadecene 0.01

8-heptadecene 0.01

9-tricosene, $(Z)-\quad 0.02$

chamazulene 0.03

9-octadecene, $(E)$ - $\quad 0.01$

1,4,5,8-Tetramethylnaphthalene $\quad 0.02$

3-octadecene, $(E)$ - $\quad 0.03$

heptacos-1-ene 0.01

Z-5-nonadecene 0.02

1,6-dimethyl-4-ethylnaphthalene (norcadalene) $\quad 0.01$

diphenylacetylene 0.01

anthracene 0.01

3-eicosene, $(E)$ - $\quad 0.04$

11-tricosene 0.02

subtotal $\quad 80.48$

polycyclic aromatic hydrocarbons (PAHs) and derivatives

naphthalene, 1,2,3,4-tetrahydro-1,8-dimethyl- 0.03

naphthalene 0.48

naphthalene, 1,2,3,4-tetrahydro-5,6-dimethyl- $\quad 0.04$

naphthalene, 1,2,3,4-tetrahydro-5-methyl- $\quad 0.23$

naphthalene, 1,2-dihydro-6-methyl- $\quad 0.04$

naphthalene, 1,2-dihydro-3-methyl- $\quad 0.29$

naphthalene, 1,2,3,4-tetrahydro-1,4-dimethyl- $\quad 0.10$

naphthalene, 1,2,3,4-tetrahydro-6-methyl- $\quad 0.03$

naphthalene, 1,2,3,4-tetrahydro-6,7-dimethyl- $\quad 0.06$

naphthalene, 1,2,3,4-tetrahydro-1,6,8-trimethyl- $\quad 0.03$

naphthalene, 5-ethyl-1,2,3,4-tetrahydro- $\quad 0.04$ 
naphthalene, 1,2,3,4-tetrahydro-2,7-dimethyl- $\quad 0.11$

naphthalene, 1,2,3,4-tetrahydro-5,7-dimethyl- $\quad 0.06$

naphthalene, 1,2,3,4-tetrahydro-1,5-dimethyl- $\quad 0.02$

naphthalene, 2-methyl- $\quad 1.19$

naphthalene, 1-methyl- $\quad 0.24$

naphthalene, 1,2,3,4-tetrahydro-1,5,7-trimethyl- $\quad 0.03$

naphthalene, 1,2,3,4-tetrahydro-1,4,6-trimethyl- $\quad 0.03$

naphthalene, 1,2,3,4-tetrahydro-6-propyl- $\quad 0.10$

naphthalene, 1,2,3,4-tetrahydro-1,6,8-trimethyl- $\quad 0.04$

naphthalene, 2-ethyl- $\quad 0.20$

naphthalene, 2,7-dimethyl- $\quad 0.61$

naphthalene, 2,6-dimethyl- $\quad 0.19$

naphthalene, 1,5-dimethyl- $\quad 0.11$

naphthalene, 1,6-dimethyl- $\quad 0.03$

naphthalene, 1,7-dimethyl- $\quad 0.03$

naphthalene, 1,6,7-trimethyl- $\quad 0.16$

naphthalene, 2,3,6-trimethyl- $\quad 0.03$

naphthalene, 1,4,6-trimethyl- $\quad 0.02$

naphthalene, 1,4,5-trimethyl- $\quad 0.03$

naphthalene, 2-(1-methylethyl)- $\quad 0.01$

naphthalene, 2-methyl-1-propyl- $\quad 0.09$

naphthalene, 2-(2-nitro-2-propenyl)- $\quad 0.01$

naphthalene, 1,2,3,4-tetramethyl- 0.01

naphthalene, 1-methyl-7-(1-methylethyl)- $\quad 0.02$

\begin{tabular}{ll}
\hline subtotal & 4.69 \\
\hline total & 100 \\
\hline
\end{tabular}

